\title{
Intraductal Papillary Neoplasm of the Bile Ducts: Case Reports with Review of the Literature
}

\author{
Dayananda Lingegowda ${ }^{1, \odot}$ Anisha Gehani ${ }^{1}$ Sumit Mukhopadhyay ${ }^{1}$ Divya Midha \\ Sudeep Banerjee ${ }^{3}$ Bharat Gupta ${ }^{1}$ \\ ${ }^{1}$ Department of Radiology, Tata Medical Center, Newtown, \\ Kolkata, India \\ 2Department of Pathology, Tata Medical Center, Newtown, Kolkata, \\ India \\ ${ }^{3}$ Department of Gastric Surgery, Tata Medical Center, Newtown, \\ Kolkata, India \\ Address for correspondence Dayananda Lingegowda, MBBS, DNB, \\ Department of Radiology, Tata Medical Center, 14 Arterial Road, \\ Newtown, Kolkata, 700160, India (e-mail: dayanandal@gmail.com).
}

J Gastrointestinal Abdominal Radiol ISGAR 2021;4:58-61.

\author{
Abstract \\ Keywords \\ - Intraductal papillary \\ neoplasm of the bile \\ duct \\ - biliary duct tumors
}

Intraductal papillary neoplasm of the bile duct (IPNB) is a rare variant of bile duct tumors, which is a counterpart of pancreatic intraductal papillary mucinous neoplasm (IPMN). These tumors need to be differentiated from other common bile duct tumors such as cholangiocarcinoma, as IPNB carries a better prognosis. A combination of enhancing intraluminal papillary filling defect, demonstrating washout and associated upstream and downstream biliary dilation, should raise the suspicion of IPNB.

\section{Introduction}

Intraductal papillary neoplasm of the bile duct (IPNB) is a rare tumor of bile duct characterized by papillary or villous growth within the bile duct lumen over fibrovascular cores. Growths are usually multifocal, with or without macroscopically visible mucin secretion. ${ }^{1}$

Previously these were identified under various names such as biliary papillomatosis, mucin-producing cholangiocarcinoma, mucin-hypersecreting bile duct tumor, and biliary intraductal papillary mucinous neoplasm (IPMN). Zen et al proposed that these tumors may belong to a single entity named IPNB. ${ }^{2}$ IPNB was adopted in the 2010 World Health Organization classification as a distinct clinical and pathologic entity. ${ }^{3}$ It is important to differentiate this entity from cholangiocarcinoma, as the prognosis of IPNB is better than that of cholangiocarcinoma.

We herein report two cases of IPMN. The first case was radiologically reported as intraductal cholangiocarcinoma, which turned out to be IPNB on histopathological evaluation. Being fully aware of the entity and its radiological features, the second case was preoperatively diagnosed as IPNB, which was confirmed on histopathology.

published online

September 1, 2020
DOI https://doi.org/

$10.1055 / \mathrm{s}-0040-1715777$

ISSN 2581-9933.

\section{Case Report}

\section{Case 1}

A 55-year-old male presented with complaints of jaundice since 3 months with intermittent fever and itching for 2 weeks.His laboratory tests demonstrated a total bilirubin of $12.4 \mathrm{mg} / \mathrm{dL}$, direct bilirubin of $7.2 \mathrm{mg} / \mathrm{dL}$, alkaline phosphatase of $490 \mathrm{UI} / \mathrm{L}$, aspartate aminotransferase (AST) of $122 \mathrm{UI} / \mathrm{L}$, alanine aminotransferase (ALT) of $88 \mathrm{UI} / \mathrm{L}$, and carbohydrate 19-9 antigen (CA19-9) of $439 \mathrm{U} / \mathrm{mL}$.Serology was negative for hepatitis $\mathrm{A}, \mathrm{B}$, and $\mathrm{C}$ infection.

Contrast-enhanced magnetic resonance imaging (MRI) of the upper abdomen along with computed tomography (CT) sections of the region of interest wasperformed, which showed enhancing intraductal soft tissue density lesion involving proximal common bile duct (CBD), common hepatic duct (CHD), primary confluence predominantly extending along the left hepatic duct (-Figs. 1 and $\mathbf{4 A - C}$ ). The lesion showed arterial contrast enhancement and washout in the venous phase. Dilated left intrahepatic biliary radicles were noted along with atrophy of the left lobe of the liver. The gallbladder was seen separate from the lesion. On the basis of the aforementioned imaging findings, a differential diagnosis of intraductal infiltrating
(C2020. Indian Society of Gastrointestinal and Abdominal Radiology. This is an open access article published by Thieme under the terms of the Creative Commons Attribution-NonDerivative-NonCommercial-License, permitting copying and reproduction so long as the original work is given appropriate credit. Contents may not be used for commercial purposes, or adapted, remixed, transformed or built upon. (https://creativecommons.org/licenses/by-nc-nd/4.0/).

Thieme Medical and Scientific Publishers Pvt. Ltd. A-12, 2nd Floor, Sector 2, Noida-201301 UP, India 


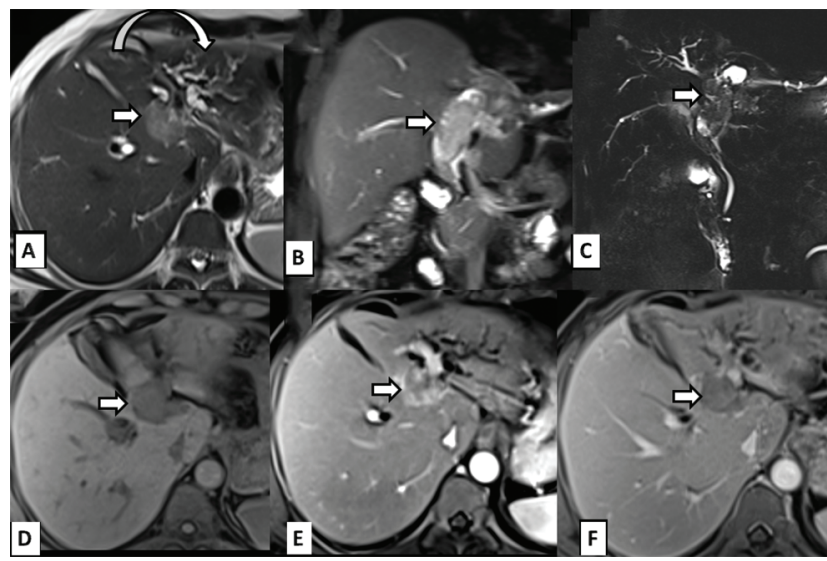

Fig. 1 Case 1: magnetic resonance imaging (MRI) images of intraductal papillary mucinous neoplasm. (A-C) T2-weighted axial, coronal, and magnetic resonance cholangiopancreatography images showing intraluminal lesions filling and expanding the left duct (arrows). Atrophy of the left lobe with left lobar ductal dilation is noted (curved arrow). (D-F) Precontrast, postcontrast arterial and postcontrast venous phase T1-weighted images showing early contrast enhancement and washout.

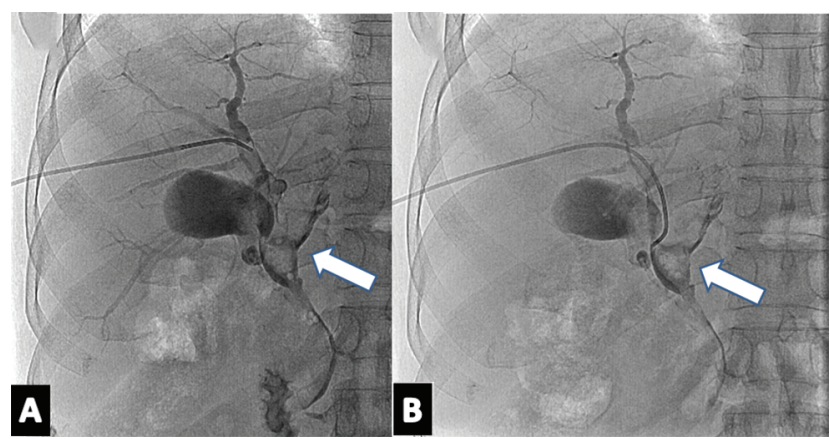

Fig. 2 (A,B) Percutaneous cholangiogram showing the filling defects at primary confluence extending into left ducts (arrows).

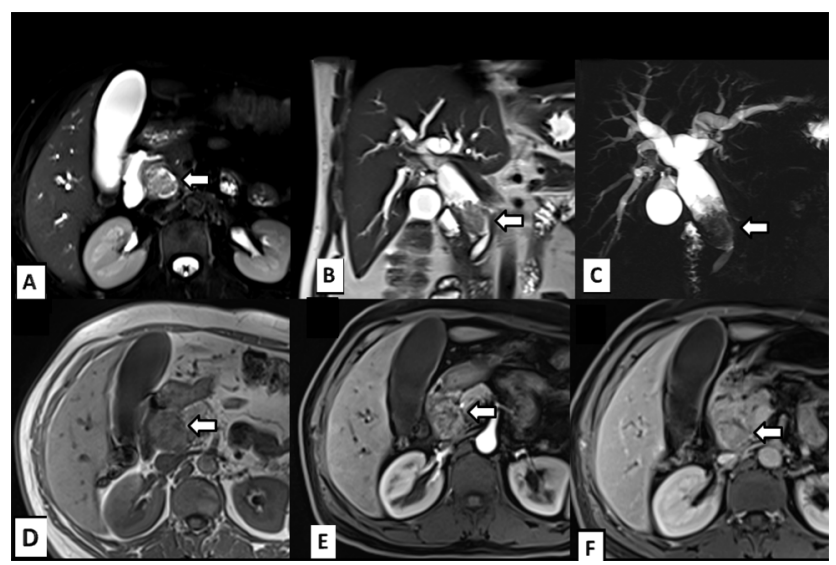

Fig. 3 Case 2: magnetic resonance imaging (MRI) images of intraductal papillary mucinous neoplasm. (A-C) T2-weighted axial, coronal, and magnetic resonance cholangiopancreatography images showing intraluminal distal common bile duct lesion(arrows). (D-F) Noncontrast, postcontrast arterial and postcontrast delayed T1-weighted images showing early contrast enhancement.

cholangiocarcinoma was given. The patient underwent right percutaneous biliary drainage (PTBD) ( - Fig. 2). Cholangiogram during PTBD showed filling defects predominantly at the

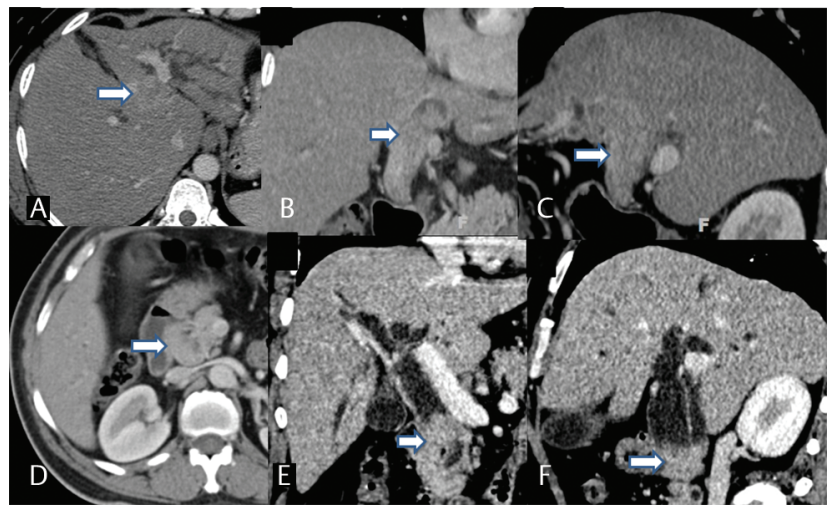

Fig. 4 (A-C) Case 1: axial, coronal, and sagittal computed tomography (CT) images showing enhancing lesions of the left duct extending into the common hepatic duct. Left intra-hepatic biliary radicals are dilated. (D-F) Case 2: axial, coronal and sagittal CT images showing enhancing lesions of the distal common bile duct causing upstream dilation.

hilum and in the left ductal system. Post-PTBD, there was a significant improvement in liver function tests. The patient underwent a left hepatectomy and caudate lobe excision with cholecystectomy.

Histopathological examination of the operative specimen was reported as intraductal papillary neoplasm with highgrade dysplasia.

\section{Case 2}

A 48-year-old male presented with complaints of intermittent fever, itching, generalized abdominal pain, and icterus for 1 month. An ultrasonography (USG) of the abdomen performed at another hospital showed soft tissue mass in the distal CBD with dilated extra and intrahepatic biliary radicles.

His laboratory tests demonstrated a total bilirubin of $22.2 \mathrm{mg} / \mathrm{dL}$, direct bilirubin of $13.6 \mathrm{mg} / \mathrm{dL}$, alkaline phosphatase of $440 \mathrm{U} / \mathrm{L}$, AST of $68 \mathrm{U} / \mathrm{L}$, and ALT of $20 \mathrm{U} / \mathrm{L}$. Serology was negative for hepatitis A, B, and C infection. Contrast-enhanced MRI of the upper abdomen along with CT sections of the region of interest showed intraductal polypoidal soft tissue density mass lesion with expansion of the middle and distal CBD ( - Figs. 3 and $4 \mathbf{D}-\mathbf{F}$ ). There was associated marked dilatation of proximal CBD, CHD, and intrahepatic biliary radicles. The periampullary region and main pancreatic duct appeared uninvolved. No definite periductal or extraductal infiltration was seen. The gallbladder and pancreas appeared normal. On the basis of the aforementioned findings and our experience with the previous case, differential diagnosis of IPNB was made and a second less likely differential diagnosis of intraductal infiltrating cholangiocarcinoma was given. The patient underwent endoscopic retrograde cholangiopancreatography (ERCP) with biliary stenting to relieve the obstructive jaundice followed by a classical Whipple's procedure. Cholangiogram performed during the ERCP showed irregular filling defects in the middle and distal CBD.

Gross examination of the pathological specimen revealed a polypoidal mass lesion along the mucosa of the distal CBD extending to involve the insertion of the 
cystic duct. The tumor was confined to the CBD without extension beyond the bile duct. Microscopic examination showed adenocarcinoma with mucinous component, arising from IPNB.

Both our patients are alive and on routine follow-up4 years after their surgery and diagnosis.

\section{Discussion}

Most of the IPNBs reported in the literature are from East Asian countries, where hepatolithiasis and clonorchiasis are endemic. ${ }^{1,4}$ IPNBs comprise 9 to $38 \%$ of all bile duct malignancies. Most patients are between 50 and 70 years of age, with slight male predominance. ${ }^{5}$ Patients usually present with intermittent abdominal pain and jaundice. IPNB is considered to have a high potential for malignancy since 40 to $80 \%$ of IPNBs are associated with invasive carcinoma or tubular or mucinous adenocarcinoma. ${ }^{6}$

IPNBs arise from biliary tree stem/progenitor cells (BTSCs) located in the peribiliary glands. ${ }^{7,8}$ In response to risk factors such as inflammation, BTSCs might undergo a series of genetic changes and progress from dysplasia to invasive carcinoma. IPNB tumor cells retain their biliary immunophenotype and obtain intestinal and gastric immunophenotypes during the evolution to carcinogenesis. Hence, almost all IPNBs express CK7, CK20, and mucin (MUC)5AC, which are markers of biliary, intestinal, and gastric epithelium, respectively. ${ }^{1,4}$ Using hematoxylin and eosin staining and immunohistochemical profiling of the mucin core proteins, four subtypes of IPNBs are identified:pancreaticobiliary, intestinal, gastric, and oncocytic. . $^{1,4}$

IPNBs most commonly present with right hypochondriac pain,cholangitis (5-59\%), andobstructive jaundice. ${ }^{6}$ Obstruction in case of IPNB can be due to tumor emboli, biliary stones, and macroscopic mucin hypersecretion. An elevated carcinoembryonic antigen (CEA) level and CA19-9 may be observed. ${ }^{9}$

There are a few similarities between IPNB and IPMN. ${ }^{1,10}$ In both entities, it has been observed that neoplasms arise within the ductal system and show a predominant intraductal growth pattern macroscopically. Microscopically, they show papillary proliferation with delicate fibrovascular cores and four types of tumor cells. Both exhibit a malignant potential to develop tubular adenocarcinoma and mucinous carcinoma. IPNBs and IPMNs have favorable biological behaviors and clinical outcomes. Mucin is macroscopically identifiable in most cases of IPMNs but only in one-third of IPNB cases.Based on these similarities, IPNB is recognized as a biliary counterpart of IPMN. ${ }^{1,7,10}$

\section{Common Imaging Finding}

Proximal bile duct dilation with intraductal masses is the most common abnormality in imaging. In some cases, both proximal and distal bile duct dilations are also observed. Five imaging patterns can be observed in IPNB., ${ }^{411}$ The most common is type 1 , which shows diffuse duct dilation with a grossly visible intraductal mass (-Fig. 5). The second common pattern is type 2 , which shows diffuse and marked duct ectasia without a grossly visible mass. Type 3 shows an intraductal

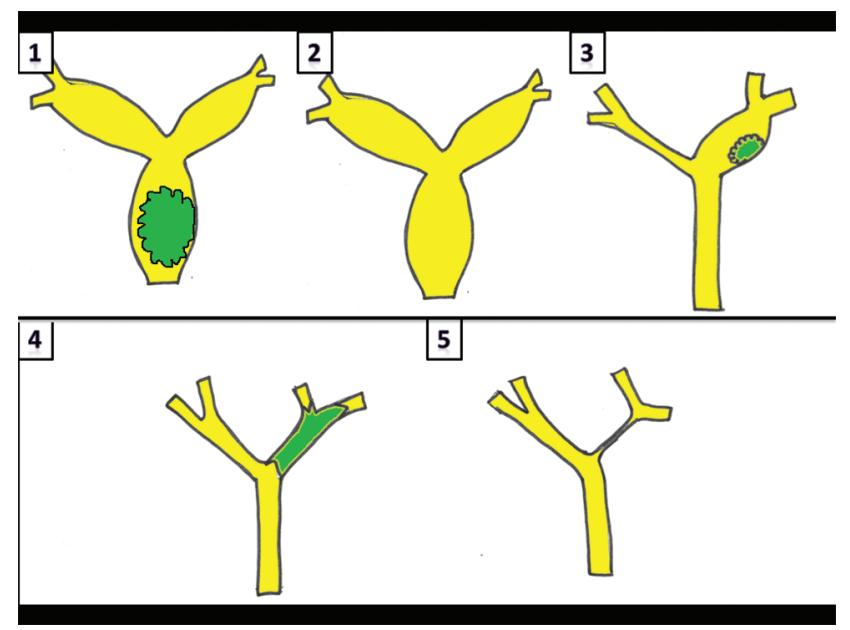

Fig. 5 Five imaging patterns of intraductal papillary neoplasm of the bile duct (IPNB). Type 1: diffuse duct ectasia with an intraluminal papillary mass. Type 2: diffuse duct ectasia without obvious visible mass. Type 3: an intraductal polypoid mass within localized duct dilation. Type 4: intraductal castlike lesions filling the lumen. Type 5: a focal stricturelike lesion with proximal ductal dilation.

papillary mass causing localized duct dilation. The least common pattern is type 4 , in which mild ductal dilation filled with intraductal castlike lesions are observed. Type 5 shows a focal stricturelike lesion with mild proximal duct dilation.

IPNB can mimic cholangiocarcinoma, intrahepatic/ extrahepatic biliary stones, Caroli's disease, choledochal cyst, recurrent pyogenic cholangitis, or primary sclerosing cholangitis.

\section{Ultrasonography}

Though the bile duct dilation is easy to detect on USG, low-echoic IPMN masses are observed in $41.2 \%$ of cases. The echogenicity of mucin is similar to that of bile. Endoscopic USG and intraductal USG are used to detect the superficial spread of tumor. ${ }^{12,13}$

\section{Computed Tomography and Magnetic Resonance Imaging}

CT can detect dilated bile ducts and tumors larger than $1 \mathrm{~cm}$. A typical MRI protocol for the assessment of biliary lesions encompasses magnetic resonance cholangiopancreatography (MRCP), conventional T1- and T2-weighted sequences diffusion-weighted imaging (DWI), and dynamic contrast-enhanced (DCE) imaging. On MRI, IPNBs appear hypointense to hepatic parenchyma on T1-weighted images and just mildly hyperintense on T2-weighted images. IPNBs often show early washout rather than the gradually persistent or progressive enhancement observed in cholangiocarcinomas. ${ }^{14}$ Neither CT nor MRI can detect the presence of mucin.

\section{Cholangiography}

MRCP, ERCP, and percutaneous transhepaticcholangiopancreatography can demonstrate characteristic diffusebile duct dilation with amorphous filling defects. On ERCP, mucin draining through the ampulla and a patulous 
ampulla is the characteristic finding. ${ }^{5}$ If ERCP is combined with cholangioscopy, evaluation of the extent of disease and obtaining the specimen for histology examination are possible.

Thus, on imaging, a combination of enhancing intraluminal papillary filling defect, demonstrating washout and associated upstream and downstream biliary dilation, should raise the suspicion of IPNB. Visualization of mucin on ERCP can be an important factor supporting the diagnosis.

Surgical resection is the treatment of choice in nonmetastatic disease, which includes segmental resection, hemihepatectomy, bile duct resection, lymph node dissection, or liver transplantation, depending on preoperative findings. ${ }^{1}$ Patients unfit for surgery can be offered palliative treatments including chemotherapy, laser ablation, iridium-192 intraluminal therapy, and PTBD.

Radiological diagnosis of IPNB is still challenging despite advances in imaging techniques. There should be a higher degree of suspicion in cases with features mentioned previously, and preoperative diagnosis should be attempted using cytology and molecular techniques.

\section{Funding}

None.

\section{Conflict of Interest}

None declared.

\section{References}

1 Wan XS, Xu YY, Qian JY, et al. Intraductal papillary neoplasm of the bile duct. World J Gastroenterol 2013;19(46):8595-8604

2 Zen Y, Fujii T, Itatsu K, et al. Biliary papillary tumors share pathological features with intraductal papillary mucinous neoplasm of the pancreas. Hepatology 2006;44(5):1333-1343

3 Bosman FT, Carneiro F, Hruban RH, Theise ND. WHO classification of tumours of the digestive system. 2010. Available at: https://www.cabdirect.org/cabdirect/abstract/20113051318
4 Kim KM, Lee JK, Shin JU, et al. Clinicopathologic features of intraductal papillary neoplasm of the bile duct according to histologic subtype. Am J Gastroenterol 2012;107(1):118-125

5 Yeh TS, Tseng JH, Chiu CT, et al. Cholangiographic spectrum of intraductal papillary mucinous neoplasm of the bile ducts. Ann Surg 2006;244(2):248-253

6 Rocha FG, Lee H, Katabi N, et al. Intraductal papillary neoplasm of the bile duct: a biliary equivalent to intraductal papillary mucinous neoplasm of the pancreas? Hepatology 2012;56(4):1352-1360

7 Nakanuma Y, Sato Y. Cystic and papillary neoplasm involving peribiliary glands: a biliary counterpart of branch-type intraductal papillary mucinous [corrected] neoplasm? Hepatology 2012;55(6):2040-2041

8 Cardinale V, Wang Y, Carpino G, Reid LM, Gaudio E, Alvaro D. Mucin-producing cholangiocarcinoma might derive from biliary tree stem/progenitor cells located in peribiliary glands. Hepatology 2012;55(6):2041-2042

9 Mann DV, Edwards R, Ho S, Lau WY, Glazer G. Elevated tumour marker CA19-9: clinical interpretation and influence of obstructive jaundice. Eur J SurgOncol 2000;26(5):474-479

10 Minagawa N, Sato N, Mori Y, Tamura T, Higure A, Yamaguchi K. A comparison between intraductal papillary neoplasms of the biliary tract (BT-IPMNs) and intraductal papillary mucinous neoplasms of the pancreas (P-IPMNs) reveals distinct clinical manifestations and outcomes. Eur J SurgOncol 2013;39(6):554-558

11 Chung YE, Kim M-J, Park YN, et al. Varying appearances of cholangiocarcinoma: radiologic-pathologic correlation. Radiographics 2009;29(3):683-700

12 Takanami K, Yamada T, Tsuda M, et al. Intraductal papillary mucininous neoplasm of the bile ducts: multimodality assessment with pathologic correlation. Abdom Imaging 2011;36(4):447-456

13 Tsuyuguchi T, Sakai Y, Sugiyama H, et al. Endoscopic diagnosis of intraductal papillary mucinous neoplasm of the bile duct. J Hepatobiliary PancreatSci 2010;17(3):230-235

14 Han JK, Lee JM. Intrahepatic intraductal cholangiocarcinoma. Abdom Imaging 2004;29(5):558-564 